4 Visser S, Bouman TK. The treatment of hypochondriasis: exposure plus response prevention vs cognitive therapy. Behav Res Ther 2001; 39: 423-42.

5 Looper KJ, Kirmayer LJ. Behavioral medicine approaches to somatoform disorders. J Consult Clin Psychol 2002; 70: 810-27.

Itoro I. Udo, specialty registrar, Jagannatha Reddy, specialty registrar, Mark Appleton, consultant psychiatrist, Lanchester Road Hospital, Tees, Esk, Wear Valleys NHS Foundation Trust, Email: itoroime.udo@tewv.nhs.uk

doi: 10.1192/bjp.199.3.250a
1 Annals of Internal Medicine. Information for authors: manuscript preparation. American College of Physicians, 2010 (http://www.annals.org/site/misc/ ifora.xhtml).

2 Dimidjian S, Davis KJ. Newer variations of cognitive-behavioral therapy: Behavioral activation and mindfulness-based cognitive therapy. Curr Psychiatry Rep 2009; 11: 453-8.

Erik Hedman, licensed psychologist, Karolinska Institutet, Department of Clinica Neuroscience, Sweden. Email: erik.hedman@sll.se

doi: 10.1192/bjp.199.3.251
Author's reply: There were no statistically significant differences between the groups at pre-treatment (as can be read from Table 2, means and standard deviations were very similar across groups). However, for several reasons we found it appropriate not to report $P$-values of baseline data. Analyses were conducted using ANCOVAs, holding pre-treatment values as covariates. Moreover, when $n$ is small, considerable variation between groups can be the case without reaching statistical significance, because of limited power. Consequently, several scientific journals (e.g. Annals of Internal Medicine ${ }^{1}$ ), advise against the use of $P$-values when comparing baseline data in randomised controlled trials.

As for the name of the treatment, we view the term internetbased cognitive-behavioural therapy (CBT) as most suitable. The treatment's theoretical foundation and its components are based on learning theory and cognitive theory. As stated in the Method and the Discussion sections, the rationale for including a mindfulness exercise was to reduce avoidance behaviours related to bodily sensations and to enhance exposure. Also, as the term CBT has been used for describing a plethora of treatments with substantial inter-treatment variability, the addition of 'modified' would probably be misleading rather than clarifying. In fact, a recent paper presents mindfulness-based cognitive therapy as 'a newer variation of cognitive behavioral therapy.'2

Regarding the control group, I agree that participating in a discussion forum hardly can be viewed as the optimal control condition. However, as the present study is the first ever to investigate internet-based CBT for health anxiety, a comparison with conventional CBT would have been premature. Such a comparison would have meant conducting a non-inferiority trial presenting difficulties regarding criteria for non-inferiority as well as the inherent assay sensitivity problem. In addition, far more participants would have needed to be randomised to internetbased CBT (because of power issues), which would have been ethically questionable. That is, far more patients would have been exposed to a potentially non-effective or even unsafe treatment. As I see it, the ideal control condition would rather have been an internet-based psychological placebo arm providing the same amount of therapist attention and treatment credibility without targeting the central proposed mechanisms of change.

When it comes to recruitment, I consider advertisements and an article in a newspaper as two quite different forms of attention. The former is under complete control of the researcher while the latter is not. As a consequence, I find it reasonable to assume that the two forms of attention have differential effects in terms of recruitment and that they therefore should be reported separately.

As for generalisability of the findings, Udo et al state that our paper tells us little as to whether internet-based CBT works in acute psychiatry settings or in an in-patient psychiatric context. I can only say that I absolutely agree. The clinic at which the present study was conducted is an out-patient clinic and internet-based CBT is not different from conventional CBT in the sense that one should be vary cautions in generalising findings from one healthcare context to another.

\section{Childhood psychotic symptoms: link between non-consensual sex and later psychosis}

Numerous studies have established a link between trauma early in life and psychosis in adulthood. ${ }^{1}$ In particular, non-consensual sex in childhood appears to robustly predict the occurrence of psychotic symptoms later in life. ${ }^{2}$ Bebbington et al ${ }^{3}$ add to this literature by demonstrating a large potential role of nonconsensual sex in the development of psychosis in a large representative sample of English adults. However, although the authors take several steps to adjust for residual confounding, they make no attempt to correct for the presence of psychotic symptoms in childhood. This is a potentially critical error as reverse causation remains a distinct possibility. Children who exhibit psychotic symptoms may be at high risk of sexual victimisation owing to their poor social skills, paucity of social relationships, and for numerous other reasons. Thus, initial mental health may explain the link between sexual abuse and adult psychosis.

In an analysis of over 3500 British adults reported elsewhere, ${ }^{4}$ I showed that non-consensual sex at age 16 or earlier placed females at a substantial risk of auditory and visual hallucinations at age $29(\mathrm{OR}=8.51,95 \% \mathrm{CI}$ 0.99-73.28). However, females who experienced hallucinations in childhood were also likely to have been forced to have sex by age 16 . When the presence of initial psychotic symptoms was taken into account the link between non-consensual sex in childhood and hallucinations in adulthood was diminished to non-significance $(\mathrm{OR}=2.43$, 95\% CI 0.09 62.88). These findings suggest that childhood sexual abuse may not be related to psychosis in adulthood over and above psychotic symptoms in childhood, at least in the domain of visual and auditory hallucinations.

Thus, when patent non-causal explanations have not been tested, vigilance is required prior to inferring that the link between sexual abuse and psychosis may be causal. Although the design utilised by Bebbington et al was cross-sectional, it would have been possible to ask participants to retrospectively gauge the age at onset of their psychotic symptoms. This would have allowed the researchers to produce a more methodologically robust assessment of the potential causal effect of sexual abuse.

Bebbington et al also identified anxiety and depression as partial mediators of the relation between sexual abuse and psychosis. However, poor initial mental health may have determined both childhood abuse and later experiences of depression, anxiety and psychosis. It is therefore of the utmost importance that those assessing the role of environmental risk factors in predicting psychosis endeavour to assess the presence of psychosis and subclinical psychotic symptoms and mental health more generally at baseline. This will allow the contribution of early environmental risk factors to psychosis to be evaluated and will provide a robust evidence base for clear policy-relevant recommendations. 
1 Read J, van Os J, Morrison AP, Ross CA. Childhood trauma, psychosis and schizophrenia: a literature review with theoretical and clinical implications. Acta Psychiatrica Scandinavica 2005; 112: 330-50.

2 Houston JE, Murphy J, Shevlin M, Adamson G. Cannabis use and psychosis: re-visiting the role of childhood trauma. Psychol Med 2011; 18 Apr. Epub ahead of print.

3 Bebbington $\mathrm{P}$, Jonas $\mathrm{S}$, Kuipers $\mathrm{E}$, King $\mathrm{M}$, Cooper $\mathrm{C}$, Brugha $\mathrm{T}$, et al. Childhood sexual abuse and psychosis: data from a cross-sectional national psychiatric survey in England. Br J Psychiatry 2011; 199: 29-37.

4 Daly M. Poor childhood mental health may explain linkages between trauma, cannabis use, and later psychotic experiences (Letter). Psychol Med 2011; 16 Jun. Epub ahead of print.

Michael Daly, lecturer in health psychology, School of Psychological Sciences, University of Manchester, UK. Email: michael.daly@manchester.ac.uk

doi: 10.1192/bjp.199.3.251a

Author's reply: Dr Daly argues that the link between child sexual abuse and adult psychosis may be the result of confounding by psychotic symptoms in childhood or adolescence. He adduces evidence for this from his secondary analysis of data from the 1970 British Birth Cohort sample. ${ }^{1}$ Of the female sample, $1.6 \%$ indicated that they had been forced to have sex by the age of 16 , and this was associated with an elevated risk of visual and auditory hallucinations at age $29(\mathrm{OR}=8.5)$. However, after controlling for the experience of such quasi-psychotic symptoms before the age of 16, the odds ratio fell to a non-significant 2.4 . Daly interprets this as indicating that this relationship exists because children with quasi-psychotic symptoms are more at risk of abuse and also at greater risk of developing psychosis as adults.

Nevertheless, Dr Daly's conclusion must equally be tentative. First, the British Birth Cohort sample apparently does not provide temporal discrimination between the occurrence of sexual abuse and the development of quasi-psychotic symptoms. Second, given that this is so, the diminution of the odds ratio after controlling for quasi-psychotic symptoms in adolescence could indicate mediation. In other words, the sexual abuse leads to adolescent symptoms which are then associated with adult symptoms. I find this explanation more plausible than the suggestion that psychotic symptoms themselves have a major effect in increasing vulnerability to abuse. There is some evidence that psychotic symptoms in adolescence are associated with prior abuse. ${ }^{2}$

It would be good to resolve this argument with appropriate data from a cohort study. However, this might not be possible: there are considerable ethical difficulties in contemporaneous enquiry about sexual abuse in child and adolescent epidemiological samples. Current research has provided some indication that the psychological consequences of abuse show similarities to psychological antecedent and maintaining factors in psychosis, ${ }^{3,4}$ and this does add plausibility to the aetiological role of sexual abuse. The particular association of early trauma with psychotic disorders (schizophreniform or bipolar) characterised by hallucinations is also difficult to explain in terms of confounding. ${ }^{5,6}$

The final worry about Dr Daly's argument is that it may detract attention from therapeutic engagement with the consequences of sexual abuse and other trauma in people with psychosis.

1 Daly M. Poor childhood mental health may explain linkages between trauma cannabis use, and later psychotic experiences (Letter). Psychol Med 2011; 16 Jun. Epub ahead of print.

2 Kelleher I, Harley M, Lynch F, Arsenault L, Fitzpatrick C, Cannon M. Associations between childhood trauma, bullying and psychotic symptoms among a school-based adolescent sample. Br J Psychiatry 2008; 193: 378-82.

3 Fowler D, Freeman D, Smith B, Kuipers E, Bebbington $\mathrm{P}$, Bashforth $\mathrm{H}$, et al The Brief Core Schema Scales (BCSS): psychometric properties and associations with paranoia and grandiosity in non-clinical and psychosis samples. Psychol Med 2006; 36: 749-59.

4 Smith B, Fowler DG, Freeman D, Bebbington $\mathrm{P}$, Bashforth $\mathrm{H}$, Garety $\mathrm{P}$, et al. Emotion and psychosis: Links between depression, self-esteem, negative schematic beliefs and delusions and hallucinations. Schizophr Res 2006; 86 $181-8$.

5 Hardy A, Fowler D, Freeman D, Smith B, Steel C, Evans J, et al. Trauma and hallucinatory experience in psychosis. J Nerv Ment Dis 2005; 193: 501-7.

6 Hammersley P, Dias A, Todd G, Bowen-Jones K, Reilly B, Bentall RP. Childhood trauma and hallucinations in bipolar affective disorder: preliminary investigation. Br J Psychiatry 2003; 182: 543-7.

Paul Bebbington, Emeritus Professor of Social and Community Psychiatry, Department of Mental Health Sciences, University College London, UK. Email: rejupbe@ucl.ac.uk

doi: 10.1192/bjp.199.3.252

\section{Revascularisation in patients with mental illness}

Mitchell et al must be congratulated on their systematic review of myocardial revascularisation in patients with mental illness. ${ }^{1}$ As physicians performing revascularisation procedures, we were disappointed by the inferior treatment received by patients with mental health problems. Fortunately, these patients account for only a minority of those presenting to acute cardiology services with symptoms and signs suggestive of acute coronary syndrome. However, when they do attend, they present cardiologists with a number of challenges, which ultimately can influence the decision regarding treatment.

Revascularisation remains an important treatment for those patients with myocardial necrosis, providing both symptomatic and prognostic benefit. ${ }^{2}$ Importantly, however, it can only be performed following invasive coronary angiography - a procedure which carries a risk of vascular complication, myocardial infarction, stroke or even death of $0.2-1.0 \%$. Clearly, patients must give appropriate consent before coronary angiography is undertaken, and this can represent an important hurdle when treating patients with mental health problems.

A second important challenge which should be considered prior to undertaking angiography, and must be considered prior to performing definitive revascularisation, is the issue of adherence to medication. Frequently, revascularisation can be performed percutaneously at the time of angiography. This procedure usually necessitates the implanting of coronary stents, which are small permanent metal scaffolds that help maintain coronary vessel patency. There are many advantages to using these devices; however, in recent times stent thrombosis has emerged as the most serious and worrying complication of their use. ${ }^{3}$ This condition is fortunately rare, but it remains a devastating, unpredictable event that has a significant morbidity and mortality; up to a third of patients will die. Research has identified that early or premature discontinuation of dual antiplatelet therapy is one of the most important risk factors in stent thrombosis. ${ }^{4}$ Consequently, cardiologists are reluctant to implant stents in patients who they feel are unlikely to comply with dual antiplatelet therapy. Unfortunately, patients with mental illness have been shown to be less adherent to medication, ${ }^{5}$ a factor which certainly has as an influence on revascularisation decisions.

These issues represent important challenges (and not excuses), which must be overcome to allow our patients to receive the most appropriate treatment. The differences in treatment certainly deserve to be highlighted and as recommended by Mitchell et al the reasons behind them require more in depth investigation, especially within the confines of the National Health Service. 